\title{
LA PARALITERATURA COMO UNA ESTRATEGIA DEL MARKETING EN EL SIGLO XXI
}

\section{PARALITERATURE AS A MARKETING STRATEGY IN THE 21ST CENTURY}

Paola Irais Mollinedo Muños*, Gladys Hernández Romero**

*Estudiante de la Licenciatura en Mercadotecnia. División Académica de Ciencias Económico Administrativas. Universidad Juárez Autónoma de Tabasco. ORCID: http://orcid.org/0000-0002-47968073.

**Doctora en Educación. Profesora Investigadora. División Académica de Ciencias Económico Administrativas. Universidad Juárez Autónoma de Tabasco. Email: doctoragladys@hotmail.com. ORCID: http://orcid.org/0000-0002-9045-2937.

Dirección para recibir correspondencia: pao_molli@hotmail.com 
LA PARALITERATURA COMO UNA ESTRATEGIA DEL MARKETING EN EL SIGLO XXI.

\section{RESUMEN}

El siglo XXI se destaca por los constantes cambios internos y externos que enfrenta una empresa frente al mercado; el latente crecimiento en el poder del consumidor crea un ambiente incierto que impacta directamente en el logro exitoso de los procesos de comunicación asertiva entre una marca y el propio consumidor. Por lo cual, se recomienda a los empresarios abordar con visión abierta a esta nueva corriente de mercadeo que determina la necesidad de reestructuración en las estrategias de marketing antes empleadas.

OBJETIVO: Proponer el estilo literario (paraliteratura), como una herramienta efectiva del marketing en comunicación de masas para el beneficio de marcas comerciales.

MATERIAL Y MÉTODO: La investigación se realizó empleando el método teórico histórico con enfoque cualitativo, haciendo provecho de fuentes de información secundarias procedentes de distintas ubicaciones geográficas que hacen testimonio al uso involuntario/inconsciente de esta herramienta y el impacto que logra como resultado.

RESULTADOS: Descubrimiento de algunos de los casos exitosos resultantes del sector editorial, los cuales propulsaron el actual posicionamiento de empresas-marcas comerciales haciendo el uso de la estructura literaria y la popularidad de los escritores en un determinado momento.

CONCLUSIONES: Los antecedentes fundamentan una aptitud latente al estilo de paraliteratura como una herramienta de marketing, puesto que el consumo de masas es un generador sin fin de ganancias y se podría considerar estar en la cúspide de impulso en pleno siglo XXI. El lector juvenil es un cliente potencial de fácil atracción mediante el consumo de material de entretenimiento donde presiden las estrategias del marketing relacional.

PALABRAS CLAVE: Paraliteratura. Mercadotecnia. Herramienta de comunicación. Publicidad. 
LA PARALITERATURA COMO UNA ESTRATEGIA DEL MARKETING EN EL SIGLO XXI.

\section{ABSTRACT}

The 21st century stands out because of the constant internal and external changes that a company faces on the market; the latent growth in the consumer power creates an uncertain environment that directly impacts on the successful achievement of the processes of assertive communication between a brand and the consumer itself. Therefore, entrepreneurs are advised to approach this new marketing trend with an open vision, which determines the need for restructuring the marketing strategies used before.

OBJECTIVE: To propose the literary style (paraliterature), as an effective tool of marketing in mass communication for the benefit of commercial trademarks.

MATERIAL AND METHOD: The research was carried out using the historical theoretical method with qualitative approach, taking advantage of secondary sources of information from different geographical locations that testify the involuntary/unconscious use of this tool and the impact that its results achieve.

RESUTS: Discovery of some of the successful cases resulting from the publishing sector which propelled the current positioning of companies-commercial trademarks making use of the literary structure and the popularity of the writers in a certain moment.

CONCLUSIONS: The antecedents support a latent paraliterature-style aptitude like a tool of marketing, since the consumption of masses is an endless generator of profits and it could be considered in the impulse peak in the 21 st century. The young reader is a potential easilyattracted customer through the consumption of entertainment material where the relational marketing strategies are the most important.

KEY WORDS: Paraliterature. Marketing. Communication tool. Advertising. 
LA PARALITERATURA COMO UNA ESTRATEGIA DEL MARKETING EN EL SIGLO XXI.

\section{INTRODUCCIÓN}

Este trabajo presenta a la literatura como una posible estrategia de marketing, donde las obras literarias clasificadas como paraliteratura, sean un medio de publicidad para el mercado comercial.

La literatura es una forma de comunicación, donde el escritor plasma sus pensamientos e ideas referentes a determinados temas pretendidos a la exposición hacia el lector. Barthes (1973) señala que: "No hay lenguaje escrito sin ostentación", para él, es posible formular una historia del lenguaje literario que no sea ni la historia de la lengua, ni la de los estilos, simplemente la historia de los signos de la literatura. El placer de escribir por el simple placer de hacerlo, el placer de leer disfrutando lo que se lee.

Para la divulgación de la lectura, se hace uso comúnmente de las funciones del marketing como medio propulsor del consumo y posicionamiento de las creaciones literarias, y el hábito de lectura, por lo cual, la relación entre ambas se identifica principalmente con la acción de un medio mercadológico hacia la literatura y no viceversa.

A partir de esta acción, señala Loaiza (2009, p. 25) en el mundo de los impresos se gesta una modernización de la esfera de la opinión pública, en la que se presentan innovaciones tecnológicas, introduciéndose un espíritu de racionalidad económica en la competición por el mercado de la opinión, es decir, por el mercado literario.

\section{MATERIAL Y MÉTODO}

La investigación está compuesta de un proceso metodológico, en el cual se muestra la evolución de la literatura, su uso con fines comerciales a nivel global y algunos de los autores influyentes de consumo; además, presenta contenido de diversas percepciones de consumidores hacia el enfoque de una literatura industrializada y la identificación de persuasión en determinadas obras literarias. 
LA PARALITERATURA COMO UNA ESTRATEGIA DEL MARKETING EN EL SIGLO XXI.

\section{MARCO TEÓRICO}

\section{La nueva era del marketing y su impacto en la empresa}

En el pasado se consideraba que para lograr el éxito en el mercado, el empresario necesitaba contar únicamente con presupuesto, materia prima y mano de obra; colocando así a la producción en masas como el objetivo central. Actualmente, la idea ha ido cambiando a causa del desarrollo en las investigaciones del marketing.

Se define al marketing como: "...proceso de planear y ejecutar la concepción, el precio, la promoción y la distribución de ideas, bienes y servicios para crear intercambios que satisfagan tanto los objetivos individuales como los organizacionales..." (AMA, 1985).

Los esfuerzos empresariales estaban enfocados en otras áreas, menos en el consumidor. Garnica y Maubert (2009) identifican seis fases de evolución - eras de orientación del marketing; donde a finales del siglo XIX, el factor primordial era la producción para luego trascender a la búsqueda de perfeccionamientos en determinadas características del artículo o servicio.

Se considera hasta este momento, que la era del marketing moderno es la última fase identificada que se encuentra en pleno desarrollo de sus fundamentos. A mitad del siglo XXI, el marketing reúne sus recursos para lanzarse en lugar de atender el mercado o sucumbir antes los competidores. Santesmases, Valderrey y Sánchez (2014) afirman que: "La mercadotecnia, por tanto, actúa fundamentalmente sobre la demanda. Identifica, crea o desarrolla demanda, (...) pero no ha de crear necesidades artificiales. Éstas deben existir bien, de forma manifiesta o latente. Han de ser reales y no aparentes".

En pleno siglo XXI, la mercadotecnia tradicional puede llegar a ser insuficiente para la búsqueda de los objetivos organizacionales. Las estrategias de marketing pasan a ser diferenciadas para cubrir las demandas homogéneas y abarcando provechosamente al público objetivo cercano. Desde los inicios del presente siglo, el marketing se ha asociado a la construcción, desarrollo y mantenimiento de vínculos e intercambios beneficiosos entre la empresa y el cliente (Wakabayashi, 2010, p. 120).

El marketing experiencial, sin duda no es la novedad de nuestro siglo, pero se considera una tendencia en el mundo de las ventas que persigue precisamente esa corriente ideológica de satisfacer al cien por ciento la demanda con un toque personalizado, que permita la conexión 
LA PARALITERATURA COMO UNA ESTRATEGIA DEL MARKETING EN EL SIGLO XXI.

cognitiva entre la experiencia de consumo y el consumidor; es decir, el marketing como elemento determinante en la formación de actitudes y comportamientos de los individuos (Buil, Martínez y Montaner, 2012).

Para Schimtt (2006) en la mercadotecnia experiencial “...los individuos son considerados sujetos que basan sus decisiones en elementos racionales, pero también, a menudo, en las emociones, las cuales despiertan una experiencia de consumo". Coloca al consumidor como un ser no sólo racional, sino capaz de ser influenciado por factores sensoriales que le orienten al nivel emocional cierta conducta o posición frente a una marca.

Moser (2012, p. 24) señala que “... la teoría detrás del marketing experiencial hace mucho énfasis en la importancia de las emociones de los consumidores"; es decir, que se basa en que los consumidores buscan transformar sus experiencias de compra, en experiencias placenteras, estimulantes y memorables.

\section{Marketing literario: El nacimiento de una literatura comercial}

A partir de la nueva orientación de mercadeo mencionada, surge la idea de conjugar el arte con el marketing, explícitamente la literatura, definida como: "Medio de tomar posición frente a los valores de la sociedad; digamos de una vez que es ideología. Toda literatura ha sido siempre ambos, arte e ideología" (Todorov, 1991).

No obstante, en el desarrollo de esta estrategia donde se plantea ejecutar una rama del arte con objetivos mercantiles, como es la literatura, múltiples percepciones difieren con la propuesta. Por lo que se considera importante resaltar que respetando el sentido lingüístico y la estética de la escritura en verso y prosa del origen literario, se recurre a la utilización del lado degradado- ya existente de la escritura que se considera una copia e insulto a la originalidad.

Entre 1860 y 1870 surge el estilo Kistch (kistchen), término de origen alemán asociado a las similitudes de pronunciación y gramática de verbos con sentidos ofensivos como: "fabricar barato", "recoger basura de la calle" y también "hacer muebles nuevos a partir de los viejos". Este estilo de expresión artística a menudo es clasificado por los grandes críticos literarios, aunque el impacto mercantil que proyecta es difícil de ignorar, ya que "...alcanzan proporciones de ventas insospechadas..." (Borque, 1971). 
LA PARALITERATURA COMO UNA ESTRATEGIA DEL MARKETING EN EL SIGLO XXI.

El estilo Kistch considera como esencial los rasgos de sustitución de lo original por otros productos que guardan algún parecido o semejanza con el producto original; sin embargo, los índices medios de lectura, compra y difusión, carecen de valor para los países y difícilmente son investigados (Barzuna, 2002). La postura sobre esta problemática desde los principios de la cúspide del estilo Kistch demuestran el bajo interés acerca del tema, según Borque (1971): “... creo que se impone urgentemente salvar esta falta, para tener un panorama objetivo del alcance cultural de los mass-media y su repercusión social."

Hacer uso del arte como un medio de comunicación o estrategia de publicidad, es un concepto ya desarrollado, persuade al público objetivo y sirve como punto de atracción (Escribano, 2010).

\section{Antecedentes de estrategias publicitarias en la literatura}

\section{Obras globales}

Las obras literarias con posición global consideradas lecturas de cultura general, son utilizadas no sólo por sus frases e imagen; también sus personajes son adoptados como recursos que proporcionan un incremento de prestigio en la marca gracias a su popularidad.

El Dr. Jekyll y Mr. Hyde, son singulares personajes literarios de la novela gótica del autor Robert Louis Stevenson, fueron utilizados como herramientas para el diseño gráfico de publicidad de la cerveza ámbar. Otro personaje literario fue "el principito", haciendo promoción para la agencia automovilística Peugeot.

Entre las obras literarias de gran impacto se encuentra la colección de 12 cuentos del escritor escocés Arthur Conan Doley, quien capturó al mercado literario con su personaje principal, el detective Sherlock Holmes, personaje que mediante su característico nivel intelectual y trama criminal fue inspiración de muchos carteles publicitarios para empresas de productos y servicios, desde comida y bebidas alcohólicas, hasta presentación y material de práctica para cursos del idioma inglés.

Son casos de éxito, que al aplicar estrategias de marketing de emociones demuestran la existencia de capacidad para construir una percepción de fidelidad en los consumidores hacia la marca o producto publicitado, todo a partir de la búsqueda de reacciones concretas provocadas por la relación literaria. 
LA PARALITERATURA COMO UNA ESTRATEGIA DEL MARKETING EN EL SIGLO XXI.

\section{Obras hispanas}

Las obras hispanas son un gran ejemplo de esta práctica publicitaria. La famosa novela del autor español Miguel de Cervantes "Don Quijote de la Mancha", ha sido una obra usada como referencia central e imagen en marcas de target principalmente masculino.

Algunos de los productos o marcas promocionados son: papel para fumar Bambú (1920), vinos finos "La Mancha" como denominación de origen Española (1932), Brandy Don Pedro (1984), carteles de promoción en el sector alimenticio y la feria del libro internacional en Cochamba (2010), la cadena de librerías Israelí y la asociación de editores de Madrid (2013); usando como lema estrofas de la obra, spots y anuncios de telefonía móvil.

Como ejemplos de este método en poesías, se encuentran en la obra "Cuándo de Chile" del poeta chileno Pablo Neruda, obra presente en la publicidad de la aerolínea española Iberia. De igual forma, el poema "Arbolé" del poeta español Pedro Calderón es constantemente presente en publicidad para promocionar marcas de aceite de oliva, incluso se pudo apreciar el uso del poema: "La vida es un sueño" del autor antes mencionado, para la campaña de marketing lanzada en un nuevo modelo vehicular de la reconocida empresa alemana Audi.

\section{La paraliteratura y el mercado}

Como éstos, existen más casos de provecho a las obras literarias. Los factores principales a reusar son: personajes, estereotipos, frases y entorno; con el objetivo de inspirar e influir una asociación nostálgica que represente el valor característico y la identificación emocional de un determinado producto o marca en el mercado.

Sin duda, las bellas artes pueden ser una oportunidad en el comercio, dando así el surgimiento del marketing cultural con estrategias como las antes mencionadas.

Gómez (2007) considera que el marketing cultural busca entretejer los objetivos del marketing con los de la cultura; es decir, a través de acciones diferentes a las convencionalmente usadas, pero muy efectivas se logra la asociación de la marca con un determinado evento. Eco (1978) retoma la clasificación entre obras literarias que cumplen con las características identificativas de la literatura y arte; puesto que una creación literaria, paraliteratura, con fines simplemente comerciales no es considerada una literatura "pura". 
LA PARALITERATURA COMO UNA ESTRATEGIA DEL MARKETING EN EL SIGLO XXI.

El autor español comparte la clasificación: "MacDonald parte de la distinción, hoy ya canónica, de los tres niveles intelectuales, high, middie y lowbrow (distinción que nos lleva a la de highbrow y lowbrow, propuesta por Van Wyck Brooks en America's Corning of Age)" (1984).

La paraliteratura puede ser nombrada con distintos términos, Jarque (1978) identifica entre ellos: literatura popular y literatura en masas; aunque entre su obra existe la discusión constante de diferenciar estos dos términos, se debe remarcar la similitud en los objetivos específicos en su producción y distribución. También se puede identificar como subliteratura, literatura industrial y literatura comercial.

La literatura popular se refiere a un tipo de lectura, cuya característica es ser aceptada por el público en general, siendo una forma extendida de cómo entretenerse, sin grandes pretensiones intelectuales. En cuanto a la literatura en masas, se caracteriza por tener un gran tiraje de ejemplares, en él se incluyen obras de autoayuda, novelas rosas, novelas históricas o novelas de fantasía épica, entre otras. Ambos términos hacen referencia al deseo que se supone plantear en este texto, independientemente del nombre, clasificación y estructura de la obra literaria según las aportaciones de autores sobre este tema, la paraliteratura puede pasar de ser considerada un simple objeto de crítica y disputa constante en el ámbito editorial, a ser de utilidad en el incremento de ganancias como medio publicitario (Morales, 2015).

Si se simplifica el proceso hasta el resultado final: un libro, ¿qué impacto tuvo? o ¿cuántos ejemplares se reprodujeron? Hablando de las creaciones hispanas, y específicamente españolas, la autora María del Socorro Tellado López, mejor conocida como Corín Tellado, fue la principal propulsora de este tipo de textos.

La industria editorial y literaria señala sus trabajos, pero la realidad consiste en el posicionamiento constante, referente a finales del siglo $\mathrm{XX}$ y principios del siglo $\mathrm{XXI}$, las estadísticas de consumo fueron sobresalientes. Sus obras se identifican de tipo "rosa", definidas como productos baratos y de entretenimiento. Las publicaciones constantes llegaron incluso a dos novelas por mes, donde cada una fue proyectada a un aproximado en venta de doscientos cincuenta mil ejemplares. Fueron cientos de páginas como oportunidad de medio publicitario igual de exitoso.

Otra autora es Agatha Mary Clarissa Miller, escritora británica creadora de novelas policiales. A nivel global, las creaciones de Agatha Christie, como es mejor conocida, impactaron en el 
LA PARALITERATURA COMO UNA ESTRATEGIA DEL MARKETING EN EL SIGLO XXI.

consumo de obras literarias y las adaptaciones cinematográficas. Sus obras El Enigmático Mr. Quin y Mrs. Marple y trece problemas, fueron ambientas en territorio español, la primera en la Isla Tenerife y la segunda en la Isla Gran Canaria.

Se considera que la publicación de ambas obras sacó a la luz al archipiélago español como destino turístico, resaltando sus vistas peculiares mundialmente y mejorando el flujo de la economía local. En 2007, se hace la publicación de la obra Crimen en el confital del autor Javier Campos Oramas, donde plantea el surgimiento de estas ideas creativas influenciadas por las Islas Canarias, también destaca la conexión entre la escritora y la historia, la apreciación obtenida del lector, la importancia de la mención de la zona geográfica con relevancia para destino de turismo y la búsqueda de información sobre la ubicación del desarrollo de estas historias posterior a su publicación (González, 2015).

Dejando a un lado la discriminación o no, de este tipo de literatura, para el marketing supone una gran oportunidad. Existe dentro de esta rama la "ventana de oportunidad" donde se debe adquirir el máximo aprovechamiento de las oportunidades en el mercado, entorno cambiante donde la diversificación es el auge del momento.

Las empresas comerciales deben emigrar a nuevos métodos que incluyan estrategias innovadoras, éstas deben adaptarse al comportamiento de los consumidores y buscar la manera más efectiva tanto de penetración como alcance. El arte es fundamental para el ser humano, es una forma libre e intrínseca de expresión, crear y compartir sentimientos plasmados en algo tangible.

Así, por medio de la literatura se practica el desarrollo de pensamientos, crear y compartir para el disfrute de la humanidad. Leer es uno de los métodos más antiguos de aprendizaje e incluso entretenimiento, la lectura es y sigue siendo resultado de una acción mercantil.

La tendencia de consumismo post-demográfico da un mayor poder de elección al consumidor y le hace partícipe potente de las decisiones de comercio en una empresa; presentando así, a un cliente que puede leer o consumir lo que objetivamente le demande su identidad y deseo.

Es casi imposible la clasificación de un lado de la literatura tan poco explorado, como es la paraliteratura. Los múltiples términos hacen difícil la integración del concepto puesto que las 
LA PARALITERATURA COMO UNA ESTRATEGIA DEL MARKETING EN EL SIGLO XXI.

percepciones son diferentes y para muchos, las características tienen líneas muy difusas con la alta literatura y para otros, no existe línea alguna.

Hasta los años 30’s, se acuñó el término Bestseller, para designar los grandes éxitos editoriales. Los géneros más frecuentados por la literatura popular son: el negro -mundo criminal-, el rosa -pasiones románticas-, el horror y la ciencia ficción, recorriendo un largo camino hasta hoy en día. Las letras respondieron a los tiempos generando unas narrativas sin más pretensión que la de entretener, centradas en el atractivo de los personajes y la trama, una literatura popular. Como resultado, se observan cuatro tipos de obras fundamentales identificadas como origen de la paraliteratura: novelas, comics, mangas y cuentos infantiles.

\section{ANÁLISIS}

En este sentido, este tipo de texto va contrario a lo que han plasmado con anterioridad otros autores en la relación de marketing-literatura. No busca desarrollar y aplicar estrategias para el impulso literario; no se pretende implementar la herramienta literaria en un mercado donde no exista el hábito de lectura; sino busca aprovechar los mercados ya existentes; es decir, el consumo de obras clasificadas como paraliteratura ofrece un amplio alcance de segmento de mercados, que han demostrado la retención y adopción de ideas que generan nuevas tendencias en la sociedad.

Su mercado principal, infantil y juvenil, son consumidores que pueden ser delicados sensorialmente en el estilo de pensamiento, incluso sus percepciones son más fáciles de modificar, y la elección de consumo va dirigida por lo que determine la opinión pública sobre los bienes de consumo y comportamientos (Sánchez, 2017).

Por lo anterior, Eco (1984) expone “... tenemos así, una situación singular: una cultura de masas en cuyo ámbito un proletariado consume modelos culturales burgueses creyéndose una expresión autónoma propia".

Un autor normalmente desarrolla la trama y los personajes según su imaginación, nada le determina esos componentes, más que el sentido y la estética deseada por él mismo. Así, para fines de marketing, se necesita interponer decisiones en la estructura total o parcial del trabajo, a ese resultado difícilmente se le consideraría una literatura alta, sin tener presente la viabilidad de participación por un autor de "alta gama" en dicha actividad. 
LA PARALITERATURA COMO UNA ESTRATEGIA DEL MARKETING EN EL SIGLO XXI.

Es aquí donde se ponderan las ventajas de la paraliteratura, detrás de su producción proporciona la oportunidad de utilizar con fines particulares la estructura de la obra, podría ser por medio de la persuasión a cierta corriente de pensamiento o tendencia, o quizá ir más allá del límite interiorizando desde la trama para dar un determinado contexto que induzca a cierta preferencia de consumo de un bien o un producto.

\section{RESULTADOS}

Tratándose de las novelas, los ejemplos pueden ser más amplios en cuanto a lo expuesto anteriormente.

Fueron las palabras redactadas por la autora estrella J. K. Rowling, junto con un toque de marketing de emociones que hechizaron al público lector a partir del año 1997. Su famosa serie literaria llamada "Harry Potter" que actualmente cuenta con una publicación de siete libros, lo que ha generado ganancias impactantes, y que en ese año eran poco imaginables para la industria editorial.

No sólo la autora se hizo acreedora de tal impacto, puesto que se beneficiaron las casas editoriales que se aventuraron en la adaptación, traducción de idiomas, producción y distribución en los diferentes continentes.

Si un millennial no ha leído uno de estos libros, visto alguna adaptación cinematográfica o escuchado de ella, no se consideraría "real millennial"; y es que ha sido considerada una de las obras que marcan principalmente la orientación de la "generación Y", adoptando un consumo masivo de los productos derivados por su creación.

El trío de jóvenes hechiceros no sólo logró ganarse el corazón de los millennials y a sus notables hábitos de consumo, sino que, presentan una amplia diversidad de público. Dando así origen a las adaptaciones cinematográficas que incrementaron aún más el grupo de consumidores Potterheads (auto-denominación popular por sus fans).

La serie de Harry Potter dio oportunidad al desarrollo de nuevos productos y servicios que, gracias a la idea creativa plasmada por la autora, la industria comercial pudo hacer realidad muchos de esos productos -inánimes, claramente- y crear su propia red de consumidores. 
LA PARALITERATURA COMO UNA ESTRATEGIA DEL MARKETING EN EL SIGLO XXI.

Actualmente, el nombre de la serie es patentado y considerado una de las marcas más productivas de la cual aún queda, comercialmente por explotar.

La publicidad referente a la serie mágica, no sólo se identifica en las adaptaciones de productos de consumo cotidiano con estampados del logo, imitaciones de las herramientas, vestuarios, figuras y uso de la imagen como referencia a la serie en servicios ofertados; también se puede identificar dentro de la misma, y es que la autora inglesa dio rienda suelta a su imaginación, haciendo mención de productos no reales, pero con altas posibilidades de serlo.

Los ejemplos más notables son la cerveza de mantequilla, bebida popular creada en la trama mágica; su constante presencia en los momentos más cúspides de la serie y la amplia gama gastronómica desarrollada en el comedor de Hogwarts, lograron despertar el deseo de llevarla a la vida real, asimismo la mención de la revista de magos "El Quisquilloso", se destacó por las publicaciones de datos infiltrados por los mismos personajes literarios y sucesos importantes; ambos productos pasaron de ser ficticios a ser productos estrellas de diferentes empresas.

La cerveza de mantequilla al no tener un método de producción real, las distintas empresas se dieron a la tarea de desarrollar sus propias recetas compitiendo unas con otras por la poción perfecta; actualmente se comercializa al rededor del mundo, y la revista fue adoptada por el fandom Potterhead quien se encarga de su creación, diseño y producción como producto "deluxe" al consumo de fans.

Siguiendo con la persuasión de consumo en novelas; un inesperado caso que atrajo a la sociedad de consumo en septiembre de este año, gracias a la producción cinematográfica elaborada por la empresa estadounidense Netflix sobre la adaptación de un Bestseller "A todos los chicos de los que me enamoré" de la autora coreano-estadounidense Jenny Han, se elevó notablemente el porcentaje de ventas del producto lácteo fermentado japonés "Yakult".

La trama de comedia y romanticismo, permitió la fácil adopción hacia sus personajes, fomentando el estilo de vida y actitud de los involucrados, el claro mercado meta eran las jóvenes y adolescentes que quedaron cautivadas con el principal personaje masculino "Peter Kavinsky" quien hace una referencia significativa en la historia con el desconocimiento y pronto gusto por la bebida láctea. 
LA PARALITERATURA COMO UNA ESTRATEGIA DEL MARKETING EN EL SIGLO XXI.

El libro presentaba con anterioridad un favorable posicionamiento en las ventas de editoriales, la inclusión de una marca quizá "involuntaria" en un producto destinado al estrellato literario, no se consideraba tan impactante hasta el caso de Yakult.

Los cambios en los porcentajes de venta del producto se hicieron notar principalmente en el mercado estadounidense, dando apertura a más puntos de distribución del mismo. El actual factor detonante, conocido como redes sociales, facilitó compartir la información mediante memes, referencias e imágenes del momento en el que el personaje lo consume. Rápidamente el mercado respondió con un grupo de incertidumbre al no conocer tan antigua marca y otro segmento atraído por nostalgia al recordar la época con la cual se sintieron identificados con el producto.

Entre las adaptaciones literarias a la pantalla con éxito comercial, se destaca Once Upon a Time, serie de televisión estadounidense estrenada en el año 2011 y finalizada a principios del 2018, creada y producida por los guionistas Adam Horowitz y Edward Kitsis. Se caracteriza por el drama y la fantasía desarrollada en cada uno de sus capítulos, la serie consiste en una trama que forma el universo de personajes procedentes de cuentos, historias de fantasía y obras literarias.

Su éxito proviene del uso estratégico de las historias más famosas como fruto de la paraliteratura, práctica de estrategia relacional de ABC. Entre ellos, se encuentran personajes de cuentos infantiles como:

Tabla 1

Once Upon a Time - cuentos infantiles

\begin{tabular}{lll}
\hline Personaje & Cuento & Autor \\
\hline Pepito el grillo & Las aventuras de Pinocho & Carlo Collidi \\
Blanca Nieves & La bella durmiente del bosque & Hermanos Grimm \\
Aurora & La bella durmiente & Charles Perrault \\
Capitán Garfio & Peter Pan y Wendy & James Matthew Barrie \\
Robin Hood & Robin Hood & Desconocido \\
Gretel & Hansel y Gretel & Hermanos Grimm \\
\hline
\end{tabular}

Fuente: Elaboración propia basada en página web ABC.com 
LA PARALITERATURA COMO UNA ESTRATEGIA DEL MARKETING EN EL SIGLO XXI.

Además, personajes de novelas reconocidas como:

Tabla 2

Once Upon a Time - Novelas literarias

\begin{tabular}{lll}
\hline Personaje & Novela & Autor \\
\hline El sombrerero loco & Las aventuras de Alicia en el País de las Maravillas & Lewis Carroll \\
Dr. Jekyll y el Sr. Hyde & El extraño caso del Dr. Jekyll y Sr. Hyde & Louis Stevenson \\
Victor Frankestein & Frankestein o el Moderno Prometeo & Mary Shelley \\
Edmond Dantés & El Conde de Montecristo & Alejandro Dumas \\
\hline
\end{tabular}

Fuente: Elaboración propia basada en página web ABC.com

Su estrategia de posicionamiento, con la utilización de personajes literarios reconocidos y adaptación de una trama mezclada entre los diferentes contextos de cada una de ellas, fue el factor que, en conjunto con la adopción de las temáticas y las réplicas de escenas originales de las obras literarias, logró la captación rápida de los televidentes; dando paso al desarrollo de siente temporadas para complacencia de los consumidores en espera del mix de personajes y trama inesperado.

Con una práctica similar la empresa global The Walt Disney Company, productora de entretenimiento con dibujos animados y producciones cinematográficas, ha sido de constante objeto de estudio para especialistas en publicidad y comunicación, que atienden a gran porcentaje de la demanda global en consumo de esparcimiento. La mayor parte de las producciones son originarias de la compañía y también hacen uso de obras de autores externos reconocidos en el mercado.

“... ¿Se tendría, por la misma razón, que tildar como propaganda todo producto cultural o mediático generado en una sociedad determinada y que refleje los valores de esa sociedad determinada?" (Pineda, s/n).

The Walt Disney Company, creadora y distribuidora de películas, cuentos infantiles y novelas que se caracteriza por la trasmisión cultural de valores, pensamientos y estilo de vida de forma persuasiva, ha sido criticada por el impacto en el comportamiento de los consumidores, su público objetivo - niños y adolescentes - son generaciones demasiado expuestas a tan gran 
LA PARALITERATURA COMO UNA ESTRATEGIA DEL MARKETING EN EL SIGLO XXI.

bombardeo de información que hace la fácil adopción de conducta y preferencias inculcadas gracias a la producción de masas a los que se encuentran rodeados.

Asimismo, las múltiples creaciones de cuentos y novelas no fueron suficientes para propagar sus objetivos e ideales, lo que le dirigió a la adquisición de la posicionada editorial productora de cómics Marvel Worlwide Inc., lo que permitió la creación y dominio su propia cultura popular, diseñada al prototipo de actitud receptora y ganando el mercado infantil en el consumo, no sólo de sus creaciones literarias y visuales. Abarca así, con mayor profundidad, y extiende su gama de productos y servicios derivados del punto principal ofertado. Además, Disney se podría empezar a considerar como un estilo de vida más que un satisfactor ocasional, dado que la influencia cognitiva da como resultado el paso a alianzas para hacer propaganda a empresas externas, corrientes de pensamiento y filosofía.

Entre diálogos, el periodista Arturo Vila preguntó al autor español Arturo Pérez-Reverte (2012), cómo se descifraba una intriga para que el lector se enganche de la primera hasta la última página de un libro, dando respuesta a tres pasos personales considerados fundamentales que pone en práctica: 1) el instinto, 2) caudal de referencia, y 3) sangre fría; reconoce también la presencia como quinto elemento general de una editorial al, Marketing.

Para el famoso escritor de paraliteratura de guerras y aventuras, la importancia en la elaboración de la obra y estudio del público determinado es fundamental: Yo creo que todos los autores, salvo los que son clásicos universales indiscutibles, tienen su momento;... si no los lees en su momento adecuado, si pasa el momento de tu vida en el que ese autor te va a dar vitaminas, lo pierdes desgraciadamente, y se aleja y nunca más coincides con él, a mí al menos es lo que me pasó (Pérez, 2012).

Con la propuesta de clasificación de Eco (1984) hacia la literatura, aconseja la identificación del tipo de estructura literaria para aprovechar al máximo el objetivo planteado al impacto en el mercado. Los Bestsellers influyeron en su inicio de lectura y desarrollo como escritor, proporcionando los puntos claves para el éxito y selección de la información necesaria en la satisfacción del lector. 
LA PARALITERATURA COMO UNA ESTRATEGIA DEL MARKETING EN EL SIGLO XXI.

\section{CONCLUSIONES}

Los constantes cambios en el sector comercial, obligan a las empresas a una adaptación de nuevos métodos, técnicas y reestructuración de modelo de negocios para tener una mejor orientación al mercado. Las estrategias de marketing tradicional se vuelven obsoletas con mayor rapidez, lo que implica la búsqueda de nuevas estrategias que hagan seguimiento de atención a la demanda presente.

El arte ha sido consecuentemente un medio de comunicación, y la literatura es una de las ramas más utilizadas con objetivos publicitarios. Los antecedentes y los recientes casos exitosos fundamentan una aptitud latente al estilo de paraliteratura como una herramienta de marketing, puesto que el consumo de masas es un generador sin fin de ganancias y se podría considerar estar en la cúspide de impulso en pleno siglo XXI. Las facilidades tecnológicas y el impulso emocional en la toma de decisión de compra, hacen posible la captación de mercados segmentados de acuerdo a los enfoques de las empresas o marcas de productos.

Un estilo de narración comercial, como es la paraliteratura, abre brecha hacia la flexibilidad del formato literario como un medio de publicidad donde se implementan estrategias de marketing con objetivo de penetración de mercado, posicionamiento de un producto o servicio y capacidad de generar relación emocional en el consumo. El lector juvenil es un cliente potencial de fácil atracción, mediante el consumo de material de entretenimiento donde presiden las estrategias del marketing relacional, creando una red de consumo traducida como un potencial éxito de ventas. 
LA PARALITERATURA COMO UNA ESTRATEGIA DEL MARKETING EN EL SIGLO XXI.

\section{REFERENCIAS BIBLIOGRÁFICAS}

American Marketing Association, AMA. (1985). AMA approves new definition. Marketing News. Recuperado de https://www.ama.org/the-definition-of-marketing/

Barthes, R. (1973). El grado cero de la escritura. México: Siglo XXI.

Barzuna, G. (2002). KISTCH: entre mercantilismo y seducción. Escena. Revista de las artes, 49-51.

Borque, J. (1971). Literatura y mass-media (Problemas de cultura de masas). Revista española De la opinión pública, (26), 45-70. doi: 10.2307/40181669

Buil, I., Martínez, E. y Montaner, T. (2012). La influencia de las acciones de marketing con causa en la actitud hacia la marca. Recuperado de https://www.sciencedirect.com/science/article/pii/S1138575812000

Eco, U. (1984). Apocalípticos e integrados en la cultura de masas, Barcelona: Lumen.

Escribano, A. (2010). Usos de la literatura en la publicidad. Recuperado de http://revistas.ucm.es/index.php/PEPU/article/view/PEPU1010220141A

García, J. (1998). La estancia de Agatha Christie en Canarias: La influencia del paisaje canario en sus novelas de intriga, Recuperado de file://C:/Users/pao_m/Downloads/La\%20estancia\%20de\%20agatha\%20christie\%20en\%2 Ocanarias.pdf

Garnica, C. y Maubert, C. (2009). Fundamentos de marketing. México: Pearson Education.

Gómez, C. (2007). Marketing cultural. Revista EAN 60, 123-146. Recuperado de https://journal.universidadean.edu.co/index.php/Revista/article/view/408/4

González, N. (2015). Agatha Christie en Canarias. Recuperado de http://aclrevistaliteraria.academiacanarialengua.org/agatha-christie-en-canarias/

Jarque, F. (diciembre, 1978). La paraliteratura: producción y consumo. Hispamérica, 7(21), 3752.

Loaiza, (2009). Colonia, Independencia y Nación Decimonónica. En Acosta, C.E., Ayala C.A. y Cruz, H. A. (Compiladores), Independencia, Independencias y espacios culturales. Diálogos de historia y literatura. 
LA PARALITERATURA COMO UNA ESTRATEGIA DEL MARKETING EN EL SIGLO XXI.

Méndez, J. (1982). Manipulación y fabricación de mitos en la subliteratura, literatura, (36), 128.

Morales, F. (2015). El éxito y los Bestsellers. Recuperado de file:///C:/Users/pao_m/Desktop/Dialnet-ElExitoYLosBestSellers-5989930.pdf

Moser, A. (2012). Estrategias de marketing experiencial aplicadas a los espacios de retail. Argentina: Universidad de San Andrés.

Pérez, A. y Vila, S. (febrero, 2012). Diálogo presentado en Bestsellers. Análisis de una literatura popular. Madrid: Fundación Juan March.

Pineda, A. (s/f). Cultura popular, cultura de masas y propaganda: límites y funciones". Sevilla: Universidad de Sevilla.

Sánchez, G. (2017). Estrategias de lectura intertextual para jóvenes en la biblioteca pública: acercamiento desde la paraliteratura hacia los clásicos literarios. Universidad de La Salle, Bogotá.

Santesmases, M., Valderrey, F. y Sánchez, A. (2014). Fundamentos de mercadotecnia. México: Grupo Editorial Patria.

Schmitt, B. H. (2006). Experiential marketing. Deusto, Barcelona.

Todorov, T. (1991). Crítica de la crítica. Paidós. Barcelona.

Wakabayashi, J. L. (2010). La investigación sobre el marketing relacional: Un análisis de contenido de la literatura 2007-2008. Recuperado de http://www.scielo.org.pe/scielo.php?pid=S2077-18862010000200008\&script 